\title{
Selection of the optimal stress test for the diagnosis of coronary artery disease
}

\author{
J A San Román, I Vilacosta, J A Castillo, M J Rollán, M Hernández, V Peral, \\ I Garcimartín, $M$ del Mar de la Torre, F Fernández-Avilés
}

Department of

Cardiology, Hospital

Universitario,

Valladolid, Spain

J A San Román

I Garcimartín

M del Mar de la Torre

F Fernández-Avilés

Department of Cardiology, Hospital Universitario San

Carlos, Madrid, Spain

I Vilacosta

Department of Nuclear Medicine, Hospital Universitario

San Carlos

M Hernández

Department of Cardiology, Hospital del Rosell, Cartagena, Spain

J A Castillo

Department of Cardiology, Hospital Río Hortega, Valladolid, Spain M J Rollán

Department of Cardiology, Hospital

Son Dureta, Palma de Mallorca, Spain

V Peral

Correspondence to: Dr J A San Román, Servicio de Cardiología, Hospital Universitario, C/ Ramón y Cajal 3, 47011 Valladolid, Spain.

Accepted for publication 22 June 1998

\begin{abstract}
Objective-To compare the value and limitations of exercise testing, dipyridamole echocardiography, dobutamineatropine echocardiography, and MIBISPECT (technetium-99m methoxyisobutyl nitrile single photon emission computed tomography) during dobutamine infusion in the diagnosis of coronary artery disease.
\end{abstract}

Design-The performance of these four tests was assessed in random order on a consecutive cohort of patients. The presence or absence of coronary artery disease was confirmed by coronary angiography. Setting-Two tertiary care and university centres.

Patients-102 consecutive patients with chest pain and no previous history of coronary artery disease. Ten patients with left bundle branch block were excluded for further analysis of exercise testing and scintigraphy results.

Results-MIBI-SPECT was the most sensitive $(87 \%)$ but the least specific test (70\%). Exercise stress testing had a sensitivity of $66 \%$, which increased to $80 \%$ when patients with inconclusive results were excluded. Dipyridamole and dobutamine echocardiography had similar sensitivity $(81 \%, 78 \%)$ and specificity $(94 \%, 88 \%)$. All four tests had similar accuracy and positive and negative predictive values. Agreement between the echocardiographic techniques was excellent (detection of coronary artery disease $87 \%, \kappa=0.72$; regional analysis $93 \%, \kappa=0.72$; diagnosis of the "culprit" vessel $95 \%, \kappa=0.92$ ), and it was good between echocardiographic techniques and MIBI-SPECT (diagnosis of the culprit vessel $90 \%, \kappa=0.84$ with dobutamine and $92 \%, \kappa=0.85$ with dipyridamole).

Conclusions-Exercise stress testing has a sensitivity comparable to other tests in patients capable of exercising and with no basal electrical abnormalities. The greatest sensitivity is offered by MIBI-SPECT and the greatest specificity is obtained with stress echocardiography. Redundant information is obtained with dipyridamole echocardiography, dobutamine echocardiography, and MIBI-SPECT. (Heart 1998;80:370-376)

Keywords: coronary artery disease; dipyridamole; dobutamine; scintigraphy
The non-invasive diagnosis of coronary artery disease is based on the detection of abnormalities in perfusion, metabolism, contractility, electrocardiography, or clinical status provoked by different types of stress. By combining a technique to detect those abnormalities with a stress agent, many types of stress test have been developed. Electrocardiographic monitoring during exercise is the most popular and widely performed of these because its indications and limitations are well known. Exercise stress testing is of no value, however, in patients who are unable to exercise, who are poorly motivated, or who have baseline electrical abnormalities.

Different techniques for the diagnosis of coronary artery disease have been compared. ${ }^{1-14}$ Nevertheless, an ideal stress test has not yet been found. Definitive conclusions cannot be drawn from these studies for the following reasons: (1) they have failed to include either exercise $4^{45-11} 1314$ or scintigraphy ${ }^{1-8}$ for comparison; (2) they have failed to consider drugs which alter oxygen supply 35111315 or demand $^{191014}$; (3) they have not specifically evaluated the implications of antianginal treatment ${ }^{1-14}$; (4) they have not examined the agreement between the tests ${ }^{1-13}$; (5) trials have been composed of heterogeneous populations including patients with previous myocardial infarction or known coronary artery disease ${ }^{1-367} ;(6)$ coronary angiography has not been performed in every patient to confirm the presence of coronary artery disease. ${ }^{14} 15$

With these considerations in mind, we undertook an investigation in which exercise stress testing, dipyridamole echocardiography, dobutamine echocardiography, and MIBISPECT (technetium-99m methoxyisobutyl nitrile single photon emission computed tomography) during dobutamine infusion, together with coronary angiography, were performed on a consecutive group of patients with chest pain and no previous history of coronary artery disease.

\section{Methods}

STUDY PATIENTS

Our population is composed of 102 consecutive patients (50 men and 52 women; mean (SD) age, 64 (10) years) with typical chest pain and no previous history of coronary artery disease. To obtain a homogeneous study group, restricted enrolment criteria had to be fulfilled. The following precluded inclusion in the study: previous myocardial infarction; Q wave on ECG; previous revascularisation; previous positive stress test (exercise, echocardiography, or scintigraphy); previous angiographically 
proven coronary artery disease; unstable angina not controlled by medical treatment; cardiac failure; congenital or valvar heart disease; cardiomyopathy.

A detailed history revealed chest pain on exertion in 14 patients, at rest in 53, and both on exertion and at rest in 35 . Though we advised that antianginal drugs should not be given, where treatment had been started by the referring physician it was continued and the tests were carried out on the same drug regimen. There were 59 patients off treatment and 43 on treatment (nine on $\beta$ blockers, 25 on calcium antagonists, and nine on both). Short acting nitrates were given as necessary. Sustained release nitrates were not used.

Tests were performed on different days and in random order within a period of seven days. Coronary angiography was performed after the tests in all cases, irrespective of the results obtained with the non-invasive techniques. No events occurred during the study period in any patient. Informed consent was obtained in each case.

EXERCISE STRESS TEST

A motor driven treadmill test was performed on all patients following a standard Bruce protocol. Blood pressure measurement and a 12 lead ECG were done before exercising and every three minutes during the test. Classic criteria for positivity were used-that is, more than $0.1 \mathrm{mV}$ of ST segment depression from baseline at 0.08 seconds from the J point.

DRUG INFUSION PROTOCOLS

A dose of $0.84 \mathrm{mg} / \mathrm{kg}$ of dipyridamole was infused over six minutes. The development of myocardial ischaemia was reversed by intravenous aminophylline $(240 \mathrm{mg}$ over one to three minutes) and glyceryl trinitrate if necessary.

Initially, dobutamine was injected at a dose of $10 \mu \mathrm{g} / \mathrm{kg} / \mathrm{min}$. Increments of $10 \mu \mathrm{g} / \mathrm{kg} / \mathrm{min}$ were given every three minutes up to a total dose of $40 \mu \mathrm{g} / \mathrm{kg} / \mathrm{min}$, which was then maintained for six minutes. At this point, atropine $(1 \mathrm{mg})$ was infused if the test was still negative and if $85 \%$ of the maximum predicted heart rate had not been reached. Propranolol (0.5$1 \mathrm{mg}$ ) was given intravenously if a positive response appeared. Intravenous glyceryl trinitrate was infused when needed.

Technetium-99m methoxyisobutyl nitrile (MIBI; $20 \mathrm{mCi}$ ) was injected one minute before cessation of the dobutamine infusion.

Situations that led to premature termination of dipyridamole or dobutamine infusion were as follows: achievement of maximal heart rate; new wall motion abnormalities; systolic blood pressure above $220 \mathrm{~mm} \mathrm{Hg}$; diastolic blood pressure above $120 \mathrm{~mm} \mathrm{Hg}$; sustained ventricular arrhythmias; symptomatic hypotension; severe angina; ST depression more than $3 \mathrm{~mm}$ or elevation more than $2 \mathrm{~mm}$.

ECHOCARDIOGRAPHIC EXAMINATION

Cross sectional echocardiographic monitoring was performed during drug infusion and up to 10 minutes after the infusion was stopped.
Commercially available machines were used. Parasternal long and short axis views and apical four and two chamber views were obtained to look for new wall motion abnormalities. Blood pressure was measured and a 12 lead ECG obtained at baseline, every three minutes during the infusion, and when required by the echocardiographer.

For purposes of analysis, the left ventricle was divided into seven segments: proximal septum, distal septum, apical, anterolateral, posterolateral, posterobasal, and diaphragmatic. This is a simplified classification based on that proposed by the American Society of Echocardiography in which the coronary anatomy is taken into account. ${ }^{16}$ Thus, both septal, apical, and anterolateral segments were assigned to the left anterior descending coronary artery, the posterolateral to the circumflex artery, and the diaphragmatic and posterobasal to the right coronary artery.

Segmental wall motion at the baseline examination was studied and graded as normal, mild hypokinesia, severe hypokinesia, akinesia, and dyskinesia. A positive response was defined as the appearance of areas of transient asynergy that were absent or of lesser degree before drug infusion. The development of dyskinesia in a previously akinetic segment was not considered a positive response but a mechanical effect. ${ }^{17}$

TECHNETIUM-99m-SESTAMIBI SPECT STUDIES One hour after injection of ${ }^{99 \mathrm{~m}}$ Tc-SESTAMIBI, tomographic imaging was performed. Resting examination was done on a different day with a second dose. A Siemens Orbiter gamma camera with a high resolution collimator was used to obtain the images. Thirty two views were collected by using a $64 \times 64$ acquisition matrix for 35 seconds each over $180^{\circ}$, from $45^{\circ}$ left posterior to $45^{\circ}$ right anterior oblique projections. Images were reconstructed using back projection with a Butterworth filter. For a better comparison of stress echocardiography and scintigraphy, scintigraphic segmentation corresponded to that used for echocardiography. Qualitative analysis was carried out and regions were classified as having normal perfusion, a stress induced perfusion defect, or a fixed perfusion defect. Both types of defects were considered positive responses for the presence of coronary artery disease.

CORONARY ANGIOGRAPHY

All patients underwent selective coronary arteriography by the Judkins technique with multiple projections. Coronary angiograms were evaluated by hand held electronic calipers. Significant coronary stenosis was considered when there was at least a $50 \%$ reduction in the luminal diameter in one or more of the major vessels or in their main branches.

\section{ANALYSIS OF TESTS RESULTS}

All tests were analysed by two independent and experienced observers who were blind to the clinical data and unaware of the results of the other tests. In cases of disagreement (exercise stress test, one case; dipyridamole echocardiography, two cases; dobutamine 
Table 1 Comparison of the different tests for the diagnosis of coronary artery disease

\begin{tabular}{|c|c|c|c|c|}
\hline \multirow[b]{2}{*}{ Performance } & \multirow{2}{*}{$\begin{array}{l}\text { Exercise stress } \\
\text { test }\end{array}$} & \multicolumn{2}{|c|}{ Echocardiography } & \multirow{2}{*}{$\begin{array}{l}\text { Scintigraphy } \\
\text { MIBI-SPECT }\end{array}$} \\
\hline & & Dipyridamole & Dobutamine & \\
\hline \multicolumn{5}{|l|}{ Sensitivity } \\
\hline Overall & $41 / 62(66 \%)$ & $54 / 66(81 \%)$ & $52 / 66(78 \%)$ & $54 / 62(87 \%)$ \\
\hline One vessel & $18 / 30(60 \%)$ & $22 / 32(68 \%)$ & $24 / 32(75 \%)$ & $24 / 30(80 \%)$ \\
\hline Multivessel & $23 / 32(71 \%)$ & $32 / 34(94 \%)$ & $28 / 34(82 \%)$ & $30 / 32(93 \%)$ \\
\hline Specificity & $24 / 30(80 \%)$ & $34 / 36(90 \%)$ & $32 / 36(88 \%)$ & $21 / 30(70 \%)$ \\
\hline Accuracy & $65 / 92(70 \%)$ & $88 / 102(86 \%)$ & $82 / 102(82 \%)$ & $75 / 92(81 \%)$ \\
\hline \multicolumn{5}{|l|}{ Predictive value } \\
\hline Positive & $41 / 45(91 \%)$ & $54 / 56(96 \%)$ & $52 / 56(92 \%)$ & $54 / 63(85 \%)$ \\
\hline Negative & $24 / 45(49 \%)$ & $34 / 46(73 \%)$ & $32 / 46(69 \%)$ & $21 / 29(72 \%)$ \\
\hline
\end{tabular}

Patients with left bundle branch block were excluded from the exercise stress test and scintigraphy analysis. sel, 24 with two vessel, and 32 with one vessel disease. Echocardiographic monitoring detected transient asynergy in 54 of the 66 during dipyridamole infusion (sensitivity $81 \%$ ) and in 52 during dobutamine-atropine infusion (sensitivity $78 \%$ ) (18 needed atropine). To analyse results of exercise testing and MIBI-SPECT, we excluded the 10 patients with left bundle branch block (four of these had coronary artery disease). Exercise stress testing was positive in 41 of the 62 patients with coronary artery disease. Sensitivity of exercise testing was therefore $66 \%$. Finally, 54 patients with coronary artery disease had perfusion abnormalities (sensitivity of MIBI-SPECT $87 \%$ ). These results show that perfusion scintigraphy was the most sensitive test ( $p=0.01$ compared with exercise test), although no statistical differences were found when compared with the echocardiographic techniques.

We studied sensitivity in several subsets of patients (table 2) in order to identify the most helpful test in a specific clinical setting. format with the possibility of frame by frame analysis. These were then analysed qualitatively by two experienced observers (both had analysed more than 200 studies).

STATISTICS

Quantitative variables are expressed as mean (SD) and were compared by the Student's $t$ test. Qualitative variables are expressed as percentages and were compared with the $\chi^{2}$ test. The Fisher test was used when indicated. Statistical significance was set at a probability (p) value of $<0.05$. A p value between 0.05 and 0.1 was considered to approach significance. Sensitivity, specificity, positive and negative predictive value, and accuracy were calculated according to standard definitions. Agreement between tests was defined as the percentage of concordant results (positive and negative). With regard to regional analysis, agreement was defined as the percentage of segments with concordant response to the drug (asynergy and perfusion defect versus normal movement and normal perfusion). Kappa ( $\kappa)$ was measured to assess the agreement excluded that expected by chance; $\kappa$ values over 0.70 were considered indicative of good agreement, those between 0.40 and 0.70 indicative of moderate agreement, and those under 0.40 of poor agreement.

\section{Results}

Overall results obtained with the different tests are shown in table 1 .

SENSITIVITY

Coronary angiography identified 66 patients with coronary artery disease: 10 with three ves-

\section{"Conclusive" exercise test}

Ten patients had left bundle branch block. Of the remaining 92 patients, 22 had an inconclusive exercise stress test. There were 16 patients who did not achieve the $85 \%$ of the maximum predicted heart rate, while basal abnormalities of ECG which did not allow interpretation were present in six. When patients with inconclusive tests were excluded from the analysis, the sensitivity of exercise testing increased to $80 \%$ (41/51 patients with disease). No statistical differences were found in the remaining tests with regard to sensitivity.

\section{SPECIFICITY}

Thirty six patients were considered not to have coronary artery disease. Six of these had left bundle branch block. Negative test results were obtained in 24 of the remaining 30 patients with exercise testing (six positives: three with ECG alterations and three with both ECG abnormalities and chest pain) and in 21 with MIBI-SPECT. Dipyridamole echocardiography was negative in 34 of the 36 patients, and dobutamine echocardiography in 32 . Thus both echocardiographic techniques had similar specificity (94\% with dipyridamole and $88 \%$ with dobutamine), and this was greater than with exercise stress testing $(80 \% ; \mathrm{p}=0.06 v$ dipyridamole test) and scintigraphy $(70 \%$; $\mathrm{p}<0.05 v$ both echocardiographic tests). The specificity of exercise stress testing and scintigraphy were similar.

Table 2 Sensitivity obtained with the different diagnostic tests according to clinical characteristics of the patients

\begin{tabular}{llllllll}
\hline & $n$ & LBBB & CAD & EST & Dipyridamole & Dobutamine & Scintigraphy \\
\hline Male & 50 & 4 & 41 & $27 / 38(71 \%)$ & $33 / 41(80 \%)$ & $31 / 41(75 \%)$ & $33 / 38(86 \%)$ \\
Female & 52 & 6 & 25 & $14 / 24(58 \%)$ & $21 / 25(84 \%)$ & $21 / 25(84 \%)$ & $21 / 24(87 \%)$ \\
$>70$ years & 30 & 6 & 21 & $10 / 19(52 \%)$ & $18 / 21(85 \%)$ & $15 / 21(71 \%)$ & $17 / 19(89 \%)$ \\
Conclusive EST ${ }^{\star}$ & 70 & - & 51 & $41 / 51(80 \%)$ & $44 / 51(86 \%)$ & $40 / 51(78 \%)$ & $44 / 51(86 \%)$ \\
LBBB & 10 & 10 & 4 & - & $2 / 4(50 \%)$ & $3 / 4(75 \%)$ & - \\
\end{tabular}

Patients with left bundle branch block (LBBB) were excluded from the exercise stress test and scintigraphy analysis.

^Negative result only accepted if at least $85 \%$ of predicted maximum heart rate achieved.

CAD, number of patients with established coronary artery disease; EST, exercise stress test; LVH, left ventricular hypertrophy detected by echocardiography, defined as thickness of interventricular septum and posterior wall $>11 \mathrm{~mm}$; $\mathrm{n}$, number of patients with clinical characteristics suggestive of coronary artery disease. 
Table 3 Haemodynamics at peak stress with the different types of stress

\begin{tabular}{llll}
\hline Variable & Exercise & Dipyridamole & Dobutamine \\
\hline Heart rate (beats/min) & $128(22)$ & $90(17)$ & $130(23)$ \\
Systolic blood pressure (mm Hg) & $172(29)$ & $110(20)$ & $146(40)$ \\
Double product & $22270(5962)$ & $10062(2926)$ & $19146(6259)$
\end{tabular}

Values are mean (SD).

${ }^{\star}$ Heart rate $\times$ systolic blood pressure.

ACCURACY AND PREDICTIVE VALUES

No statistical differences were found in comparisons of diagnostic accuracy. Positive predictive values were also similar in the different tests. Exercise testing showed a lower negative predictive value than the remaining tests, but there were no statistically significant differences between the tests.

\section{AGREEMENT BETWEEN TESTS}

For detection of presence or absence of coronary artery disease, a moderate overall agreement was obtained between exercise stress testing and pharmacological stress echocardiography: $75 \%$ with dipyridamole $(\kappa=0.49)$ and $72 \%$ with dobutamine-atropine $(\kappa=0.43)$. Scintigraphy had a $72 \%$ agreement with exercise stress testing $(\kappa=0.48), \quad 77 \%$ $(\kappa=0.53)$ with dobutamine echocardiography, and $82 \%(\kappa=0.61)$ with dipyridamole echocardiography. A good agreement was found when dipyridamole and dobutamineatropine were compared $(87 \% ; \kappa=0.72)$.

On regional analysis, there was agreement between dipyridamole and dobutamineatropine echocardiography in 644 of the 693 segments studied $(93 \% ; \kappa=0.72)$. MIBISPECT showed $86 \%$ agreement (552 segments; $\kappa=0.65$ ) with dobutamine, and $84 \%$ with dipyridamole (539 segments; $\kappa=0.63$ ). Twenty one poorly visualised segments from 17 patients (13 patients with one segment and four with two segments) were excluded from the analysis.

Finally, analysis of concordance for diagnosis of vessel disease was carried out in patients with positive echocardiographic and scintigraphic responses. Asynergies provoked by dipyridamole and dobutamine-atropine appeared in territories nourished by the same artery in $95 \%$ of cases $(\kappa=0.92)$. Regarding MIBI-SPECT, perfusion defects and asynergies were considered to be dependent on the same artery in $90 \%$ of cases with dobutamine echocardiography $(\kappa=0.84)$ and $92 \%$ with dipyridamole echocardiography $(\kappa=0.85)$.

\section{HAEMODYNAMIC RESPONSE}

Table 3 shows the heart rate, systolic blood pressure, and double product (heart rate $\times$ systolic blood pressure) achieved with the different types of stress. Results of all these variables were lower with dipyridamole than with exercise and dobutamine. The double product obtained with exercise was higher than with dobutamine because of the higher systolic blood pressure achieved. Heart rate at peak stress during exercise and dobutamine was similar.
SIDE EFFECTS

Minor side effects such as palpitations, headache, flushing, or nausea appeared in 36 patients during dipyridamole infusion (36\%) and in 35 during dobutamine infusion (35\%). Non-sustained supraventricular and ventricular arrhythmias developed more often with dobutamine than with dipyridamole $(8 \%$ v $3 \%$; $\mathrm{p}=0.07)$. The incidence of major complications was greater during dobutamine than during dipyridamole $(8 \% v 3 \% ; \mathrm{p}=0.07)$. The following were considered to be major complications: left heart failure (one patient with dobutamine, one with dipyridamole), severe symptomatic hypotension (two with dobutamine, two with dipyridamole), severe hypertension (three with dobutamine, none with dipyridamole), and sustained ventricular tachycardia (two with dobutamine, none with dipyridamole).

\section{Discussion}

This study was designed in a rigorous fashion to compare the role of different non-invasive techniques available for clinical use in the diagnosis of coronary artery disease. We therefore chose those techniques that best represent the current diagnostic armamentarium. Exercise stress testing is still considered the first step in a rational diagnostic approach. For stress echocardiography we chose dipyridamole and dobutamine because they have both been extensively validated, ${ }^{1-3}$ though it is not clear whether either is superior ${ }^{18}$ - for example, Salustri et al claimed that dipyridamole was the better of the two, ${ }^{5}$ while Beleslin et al preferred dobutamine. ${ }^{7}$ We did not include adenosine echocardiography for the following reasons: side effects are almost universal ${ }^{19}$; its diagnostic accuracy has been shown to be less than that of dobutamine ${ }^{4}{ }^{11}$; and its short half life may preclude the development of transient asynergy or its identification if it does appear. There is increasing recognition that ${ }^{99} \mathrm{~T}$ C-MIBI is an excellent perfusion marker for myocardial imaging and that SPECT offers a three dimensional reconstruction of the heart, permitting identification of perfusion heterogeneity in specific coronary regions.

Based on the pathophysiological continuum termed the "ischaemic cascade", ${ }^{20}$ perfusion techniques would detect early phases of mismatch between oxygen supply and demand, and should therefore be more sensitive than echocardiography and exercise stress testing. However, early phases of mismatch might not be accompanied by ischaemia, and so specificity should be higher with the latter approaches. Our results confirm these theoretical considerations. MIBI-SPECT was more sensitive $(87 \%)$ than dipyridamole $(80 \%)$ and dobutamine echocardiography $(78 \%)$ or exercise stress testing $(66 \%)$. On the other hand, the specificity of pharmacological stress echocardiography ( $94 \%$ for dipyridamole and $88 \%$ for dobutamine) was greater than that of scintigraphy $(70 \%)$. 
EXERCISE STRESS TEST

Exercise stress testing is adequate in patients with a normal exercise capacity and normal baseline ECGs. ${ }^{21}$ In this study, 32 patients $(32 \%)$ had an inconclusive result with exercise testing. Twenty two patients did not exercise long enough to achieve the target heart rate and 10 had left bundle branch block. In patients who exercised sufficiently and had interpretable ECGs, the sensitivity was similar to the remaining techniques. Thus exercise stress testing offers a high degree of accuracy in patients with a "conclusive" result-in other words, no further procedure is required for diagnostic purposes. It should be recognised, however, that false positive results can be encountered in women ${ }^{22}$ and in hypertensive patients. ${ }^{23}$ In such individuals, pharmacological stress echocardiography maintains high specificity. ${ }^{12} 2224$

DIPYRIDAMOLE VERSUS DOBUTAMINE

Dipyridamole and dobutamine, in combination with echocardiography, are both of value in identifying patients with ischaemic heart disease. Which of these two drugs, if either, is preferable when searching for asynergy remains to be settled, and investigators have reported differing results. ${ }^{4-818}$ Such discrepancy is likely to be related to different enrolment criteria, the use of antianginal agents, or different drug infusion protocols. The inclusion of patients with known coronary artery disease tends to overestimate the sensitivity of the test assessed $^{25}$ and can bias the results. Thus conclusions cannot be applied to a general population. It has recently been shown that the addition of atropine when a dipyridamole test is negative enhances the sensitivity of dipyridamole echocardiography without decreasing its specificity. $^{26}$

Major side effects were slightly more common with dobutamine than with dipyridamole infusion, as reported by others. ${ }^{6}$ The three most extensive studies dealing with complications during dipyridamole ${ }^{27}$ or dobutamine administration $^{28}{ }^{29}$ further extend our results. Combining the results of all these studies, dipyridamole provoked severe arrhythmias in $0.17 \%$ of patients, compared with $7 \%$ with dobutamine, while dipyridamole infusion had to be interrupted prematurely because of major side effects in only $0.6 \%$ of patients, in contrast with $7 \%$ during dobutamine.

The good agreement in detecting the presence or absence of both coronary artery disease and regional asynergy indicates that redundant rather than complementary information is provided by these two pharmacological tests; furthermore, in respect of regional analyses, the agreement was excellent not only for segments but also when the "culprit" coronary artery was considered. Our results suggest that these two tests should not both be used in the same patient for diagnostic purposes.

ECHOCARDIOGRAPHY VERSUS SCINTIGRAPHY

Stress echocardiography and scintigraphy seek different end points - ventricular asynergy and perfusion abnormalities, respectively-but with a common purpose: the identification of coronary artery disease. Patients with perfusion abnormalities (positive scintigraphy) and no ventricular asynergy (negative echocardiography) have an imbalance strong enough to create perfusion abnormalities but not to precipitate ventricular asynergy. Whether there is heterogeneity of perfusion with no ischaemia or with ischaemia that is too slight or too brief to be detected by current echocardiographic techniques is far from clear. It has recently been suggested that the presence or absence of ventricular asynergy during dobutamine infusion is not related to the extension of perfusion abnormalities. ${ }^{30}$ Other investigations, nevertheless, support the view that the development of asynergy during dobutamine infusion defines a group of patients with more severe perfusion abnormalities detected by MIBI-SPECT. ${ }^{31}$ The limitations of scintigraphic techniques in patients with hypertension and left ventricular hypertrophy ${ }^{32}$ and with left bundle branch block ${ }^{33}$ are well known. Several studies have demonstrated that stress echocardiography is to be preferred in these subgroups of patients. ${ }^{123}$

Some investigators have compared stress echocardiography with scintigraphy. ${ }^{9-13} 34$ A slightly higher sensitivity and lower specificity of scintigraphic techniques is in agreement with our experience. One study ${ }^{35}$ reported a sensitivity of $58 \%$ with dipyridamole echocardiography and $95 \%$ with dipyridamole-thallium scintigraphy. The discrepancy with the other published reports is explained by the use of a lower dose of dipyridamole $(0.56 \mathrm{mg} / \mathrm{kg})$ instead of the high dose protocol $(0.84 \mathrm{mg} / \mathrm{kg})$, which has shown that sensitivity can be increased without reducing specificity. ${ }^{1}$

A moderate concordance was found in our study when pharmacological stress echocardiography and scintigraphy were compared. The agreement was excellent with regard to the diagnosis of the "culprit" coronary lesion. Previous studies confirm our results. ${ }^{14}{ }^{15} 36$ Again, the use of both procedures in the same patient is not advised. Since stress echocardiography is highly operator dependent, ${ }^{37}$ the technique chosen should depend on the expertise of each centre in stress echocardiography and scintigraphy.

\section{LIMITATIONS}

One potential limitation of this study is that digitised imaging was not used. This allows side by side comparisons for detecting subtle wall motion abnormalities. It may be argued that the relatively low sensitivity of stress echocardiography could be explained by the qualitative nature of the analysis performed. However, the place of digital imaging in interpreting stress echocardiography is unresolved, some studies claiming that digital image processing is superior $^{38}$ while others have suggested similar ${ }^{39}$ or even higher diagnostic accuracy with traditional videotape analysis. ${ }^{40}$ Likewise, we used a semiquantitative method to score the scintigrams. It has been claimed that quantitative analysis is superior to semiquantitative analysis, but this is also controversial-for 
example, sensitivity has not been found to be dependent on the method used ${ }^{4142}$; in contrast, one study suggests a higher specificity with the quantitative approach ${ }^{41}$ while another claims lower specificity. ${ }^{42}$

We used dobutamine rather than dipyridamole for the perfusion studies. The accuracy of dipyridamole scintigraphy has been more extensively reported. Nonetheless, dobutamine infusion has been found to be as accurate as adenosine during scintigraphic studies ${ }^{11}$ and a high concordance has been shown in comparisons between dobutamine and exercise combined with perfusion techniques. ${ }^{13}$

Finally, although our study was conducted on consecutive patients, our institutions are tertiary care centres and this may have biased the results since the predictive values depend on the prevalence of the disease. Therefore, results may not be applicable to a general population.

CONCLUSIONS

The non-invasive technique of choice for the diagnosis of coronary artery disease is still to be established. Available techniques all have advantages and drawbacks and none can be considered suitable for all patients. To obtain the highest efficiency with each technique we must be conscious of its limitations. Some practical conclusions can be drawn from our study: exercise stress testing is very accurate in patients with the capacity to exercise enough to achieve a "conclusive" result; scintigraphy with MIBI-SPECT is very sensitive but less specific than pharmacological stress echocardiography; redundant information is obtained with dipyridamole echocardiography, dobutamine echocardiography, and MIBI-SPECT. We greatly appreciate the technical assistance of Josefina Albú-
jar, Olga Allfonso, Ana España, Inés Gómez, and María Sánchez.

1 Picano E, Lattanzi F, Masini $\mathrm{M}$, et al. High dose dipyridamole echocardiography test in effort angina dipyridamole echocardiography test in

pectoris. F Am Coll Cardiol 1986;8:848-54.
2 Sawada SG, Segar DS, Ryan T, et al. Echocardiographic Sawada SG, Segar DS, Ryan T, et al. Echocardiographic
detection of coronary artery disease during dobutamine detection of coronary artery disease
infusion. Circulation 1991;83:1605-14.

3 Mazeika PK, Nadazdin A, Oakley CM. Dobutamine stress echocardiography for detection and assessment of coronary artery disease. F Am Coll Cardiol 1992;19:1203-11.

4 Martin TW, Seaworth JF, Johns JP, et al. Comparison of adenosine, dipyridamole and dobutamine in stress echocardiography. Ann Intern Med 1992;116:190-6.

5 Salustri A, Fioretti PM, McNeill AJ, et al. Pharmacological stress echocardiography in the diagnosis of coronary artery disease and myocardial ischaemia: a comparison between dobutamine and dipyridamole. Eur Heart $\mathcal{F}$ 1992;13:135662.

6 Previtali M, Lanzarini L, Fetiveau R, et al. Comparison of dobutamine stress echocardiography, dipyridamole stress echocardiography and exercise stress testing for diagnosis echocardiography and exercise stress testing for diagnosis
of coronary artery disease. Am f Cardiol 1993;72:865-70.

7 Beleslin BD, Ostojic M, Stepanovic J, et al. Stress echocardiography in the detection of myocardial ischemia Head-to-head comparison of exercise, dobutamine and dipyridamole tests. Circulation 1994;90:1168-76.

8 Dagianti A, Penco M, Agati L, et al. Stress echocardiography: comparison of exercise, dipyridamole, and dobutamine in detecting and predicting the extent of coronary artery disease. 7 Am Coll Cardiol 1995;26:18-25.

9 Picano E, Parodi O, Lattanzi F, et al. Comparison of dipyridamole-echocardiography test and exercise thallium201 scanning for diagnosis of coronary artery disease. $A m \mathcal{F}$ Noninv Cardiol 1989;3:85-92.

10 Parodi O, Bissi G, Cassucci R, et al. Clinical efficacy of Tc99m-MIBI scintigraphy associated to echocardiography and dipyridamole test for the detection of coronary artery disease. A multicentre study [abstract]. Circulation 1989; 80(suppl II):II-620.

11 Marwick T, Willemart B, D'Hont AM, et al. Selection of the optimal nonexercise stress for the evaluation of ischemic regional myocardial dysfunction and malperfusion. Circulation $1993 ; 87 \cdot 345-54$

12 Marwick T, D'Hondt A, Baudhuin T, et al. Optimal use of dobutamine stress for the detection and evaluation of coronary artery disease: combination with echocardiography or scintigraphy, or both? f Am Coll Cardiol 1993;22:15967.

13 Hoffman R, Lethen H, Kleinhaus E, et al. Comparative evaluation of bicycle and dobutamine stress echocardiography with perfusion scintigraphy and bicycle electrocardiogram for identification of coronary artery disease. $A m \mathcal{F}$ Cardiol 1993;72:555-9.

14 Jain A, Suárez J, Mahmarian JJ, et al. Functional significance of myocardial perfusion induced by dipyridamole using thallium-201 single photon emission computed tomography and two-dimensional echocardiography. Am $\mathcal{F}$ Cardiol 1990;66:802-6.

15 Forster T, McNeill AJ, Salustri A, et al. Simultaneous dobutamine stress echocardiography and technetium- $99 \mathrm{~m}$ isonitrile single-photon emission computed tomography in patients with suspected coronary artery disease. $\mathcal{F}$ Am Coll Cardiol 1993;21:1591-6.

16 Segar DS, Brown SE, Sawada SG, et al. Dobutamine stress echocardiography: correlation with coronary lesion severity as determined by quantitative angiography. $\mathcal{F ~ A m ~ C o l l ~ C a r - ~}$ diol 1992;19:1197-202.

17 Arnese $\mathrm{M}$, Fioretti $\mathrm{P}$, Cornel $\mathrm{JH}$, et al. Akinesis becoming dyskinesis during high-dose dobutamine stress echocardiography: a marker of myocardial ischemia or a mechanical phenomenon? Am 7 Cardiol 1994;73:896-9.

18 San Román JA, Vilacosta I, Castillo JA, et al. Dipyridamole and dobutamine-atropine stress echocardiography in the diagnosis of coronary artery disease. Comparison with exercise stress test, analysis of agreement and impact of antianginal treatment. Chest 1996;111:1248-54.

19 Cerqueira MD, Verani MS, Schwaiger M, et al. Safety profile of adenosine stress perfusion imaging: results from the Adenoscan multicenter trial registry. $\mathcal{f} \mathrm{Am}$ Coll Cardiol 1994;23:384-9.

20 Nesto RW, Kowalchuck GJ. The ischemic cascade: temporal sequence of hemodynamic, electrocardiographic and ymptomatic expressions of ischemia. Am $\mathcal{f}$ Cardiol 1987;59:23-30C.

21 Kotler TS, Diamond GA. Exercise thallium-201 scintigraphy in the diagnosis and prognosis of coronary artery disease. Ann Intern Med 1990;113:684-702.

22 Masini M, Picano E, Lattanzi F, et al. High dose dipyridamole-echocardiography test in women: correlation with exercise-electrocardiography test and coronary arteriography. F Am Coll Cardiol 1988;12:682-5.

23 Wroblewski EM, Pearl FJ, Hammer WJ, et al. False positive stress tests due to undetected left ventricular hypertrophy. Am 7 Epidemiol 1982;115:412-17.

24 Picano E, Lucarini AR, Lattanzi F, et al. Dipyridamole echocardiography in essential hypertensive patients with chest pain. Hypertension 1988;12:238-43.

25 Armstrong WF, O'Donnell J, Ryan T, et al. Effect of prior myocardial infarction and extent and location of coronary disease on accuracy of exercise echocardiography. $7 \mathrm{Am}$ Coll Cardiol 1987;10:531-8.

26 Pingitore A, Picano E, Colosso MQ, et al. The atropine factor in pharmacological stress echocardiography. $\mathcal{f} \mathrm{Am}$ Coll Cardiol 1996;27:1164-70.

27 Picano E, Marini C, Pirelli S, on behalf of the EchoPersantine International Cooperative Study Group. Safety of intravenous high-dose dipyridamole echocardiography. Am f Cardiol 1992;70:252-8.

28 Mertes H, Sawada SG, Ryan T, et al. Symptoms, adverse effects, and complications associated with dobutamine stress echocardiography. Experience in 1118 patients. Circulation 1993;88:15-19.

29 Poldermans D, Fioretti PM, Boersma E, et al. Safety of dobutamine-atropine stress echocardiography in patients with suspected or proven coronary artery disease. $A m \mathcal{F}$ Cardiol 1994;73:456-9.

30 Elhendy A, Geleijnse ML, Roelandt JR, et al. Dobutamineinduced hypoperfusion without transient wall motion abnormalities: less severe ischemia or less severe stress? $\mathcal{F}$ Am Coll Cardiol 1996;27:323-9.

31 De la Torre MM, San Román JA, Vilacosta I, et al. Ecocardiografía-MIBI comn dobutamina: significado de las alteraciones de la contractilidad en pacientes con alteraciones de la perfusión. Rev Esp Cardiol 1998;51:204-10.

32 De Puey EG, Guertler-Krawczynska E, Perkins JW, et al. Alterations in myocardial thallium-201 distribution in patients with chronic systemic hypertension undergoing single-photon emission computed tomography. Am $\mathcal{F}$ Cardiol 1988;62:234-8.

33 De Puey EG, Guertler-Krawczynska E, Robbins WL. Thallium-201 SPECT in coronary disease patients with left bundle branch block. F Nucl Med 1988;29:1479-85.

34 Günalp B, Dokumaci B, Uyan C, et al. Value of dobutamine technetium-99m-sestamibi SPECT and echocardiography in the detection of coronary artery disease compared with coronary angiography. $\mathcal{F}$ Nucl Med 1993;34:889-94.

35 Perin EC, Moore W, Blume M, et al. Comparison of dipyridamole-echocardiography with dipyridamolethallium scintigraphy for the diagnosis of myocardial ischemia. Clin Nucl Med 1991;16:417-20.

36 Geleijnse ML, Elhendy A, van Domburg RT, et al. Cardiac imaging for risk stratification with dobutamine-atropine stress testing in patients with chest pain. Echocardiography, scintigraphy, or both? Circulation 1997;96:137-47. 
37 Picano E, Lattanzi F, Orlandini A, et al. Stress echocardiography and the human factor: the importance of being expert. I Am Coll Cardiol 1991;17:666-9.

38 Marwick T, D'Hont AM, Mairesse GH, et al. Comparative ability of dobutamine and exercise stress in inducing myocardial ischemia in active patients. Br Heart $\mathcal{F} 1994 ; 72: 31$

39 Salustri A, Fioretti PM, Pozzoli MM, et al. Dobutamine stress echocardiography: its role in the diagnosis of coronary artery disease. Eur Heart $\mathcal{F}$ 1992;13:70-7.
40 Castini D, Gentile F, Ornaghi M, et al. Dobutamine Castini D, Gentile F, Ornaghi $\mathrm{M}$, et al. Dobutamine echocardiography: usefulness

41 Mahmarian JJ, Boyce TM, Goldberg RK, et al. Quantitative exercise thallium-201 single photon emission computed tomography for the enhanced diagnosis of ischemic heart disease. F Am Coll Cardiol 1990;15:318-29.

42 Laarman GJ, Bruschke AV, Verzijlbergen JG, et al. Thallium-201 scintigraphy after dipyridamole infusion with low exercise: quantitative analysis versus visual analysis. Eur Heart f 1990;11:162-72.

\section{IMAGES IN CARDIOLOGY}

\section{Idiopathic chylopericardium}

In 1988 a 26 year old man came to our department because of cardiomegaly found at a routine chest $x$ ray. He had no history of cardiac disease and was asymptomatic. Basic electrocardiography was normal. Echocardiography revealed normal cardiac chambers and valves and a discrete pericardial effusion; therefore, the patient underwent diagnostic pericardiocentesis. The fluid was milky with a high cholesterol, triglyceride, and protein content $(1.68 \mathrm{mmol} / \mathrm{l}, 48.58 \mathrm{mmol} / \mathrm{l}$, and $50.0 \mathrm{~g} / \mathrm{l}$, respectively), while a large chylomicron band was present at electrophoresis. The presence of lung or mediastinum abnormalities was excluded by thoracic computed tomography. A lymphangiography showed an open thoracic ductus as far as the fifth thoracic vertebra, then the contrast spread towards the anterosuperior portion of the thoracic wall, shaping small sacculations and thin lymphatic ways. Thus, an idiopathic chylopericardium owing to mediastinal lymphangiectasias was diagnosed. The patient was kept on a medium chain triglyceride diet and was not surgically treated. At nine years' follow up the pericardial effusion had completely disappeared.

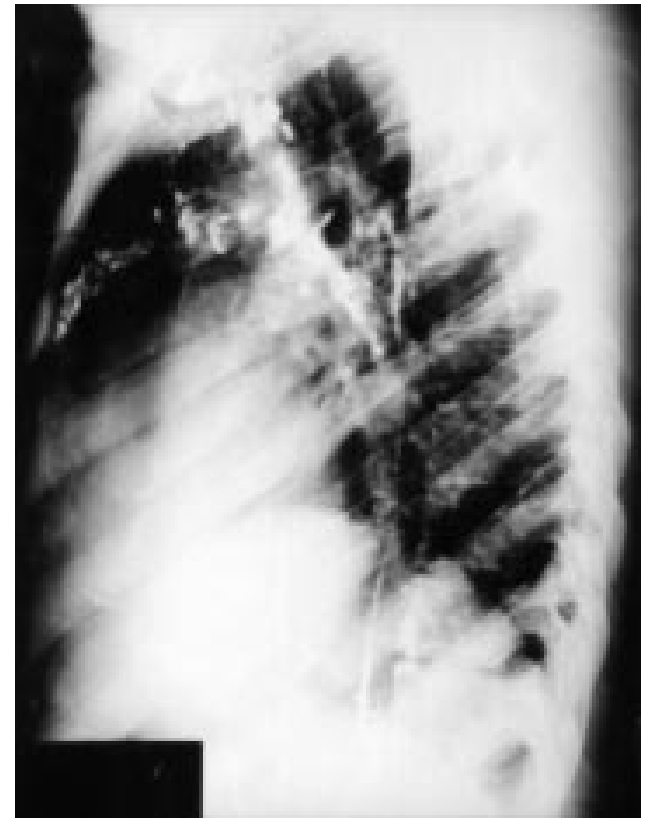

MARIA CRISTINA BARATELLA MASSIMO MONTESELLO PIERGIORGIO MARINATO 